\title{
HIPPARCOS AND REFERENCE SYSTEMS
}

\author{
F. MIGNARD \\ O.C.A. / CERGA \\ Av. Copernic \\ 06130 Grasse (France)
}

\begin{abstract}
.
The Sphere determined from the observations carried out by the ESA satellite Hipparcos will have a residual rotation with respect to distant extragalactic sources. The alignement of the two systems of reference will be made at the mission completion. The procedure to be applied is discussed along with its expected accuracy.
\end{abstract}

\section{INTRODUCTION}

The main objective of global astrometry is to build a reference system materialized by the position and proper motion of as many luminous sources as possible (Kovalevsky, 1984). HIPPARCOS will provide such a system through the sphere solution. The HIPPARCOS solution will ultimately give for each star

i) a position as seen from the barycenter of the solar system at a reference epoch to ( for example 1991 January 1, 12h TB )

ii) the components of the proper motion at the same epoch. For a typical Hipparcos star $(V=9 \mathrm{mag})$ it is expected that the positions at the epoch and proper motion will be determined respectively with an r.m.s. error smaller than 2 mas and 2 mas per year, provided the mission lifetime is at least 3 years.

\section{THE HIPPARCOS AND VLBI REFERENCE FRAMES}

However the Hipparcos measuring system will only provide a perfectly consistent system of angular arcs between the $\sim 115000$ entries of its stellar catalogue and not their absolute position on the sky. The mathematical structure of the equations relating the photons counts 
recorded by the detectors to the astronomical coordinates of the stars are invariant by any 3-D rotation. Accordingly two catalogues differing by a mere rotation are equally acceptable solutions of the observation equations. Thus an arbitrary convention is necessary to finalize the Hipparcos catalogue. Put in other words the normal equations possess a global symmetry which let them unchanged by any transformation of the group $\mathrm{SO}(3)$, and so are the solutions of these equations. From a more physical point of view one can say that Hipparcos fits a very large number of arcs drawn on a sphere between more than 100000 points. The relative position of the points does not depend on the precise orientation of the sphere, which therefore will have to be arbitrarily fixed at a later stage.

A question arises naturally : among all the possible conventions to define the coordinate system for the Hipparcos catalogue, are there some more compelling than others? If stars were only used as milestones to track the displacement of moving objects, without reference to their dynamics, taking one convention rather than another would not make any difference. But since the time of Newton, physicists and astronomers have singled out the inertial systems, with respect to which the laws of nature look more simple. Thus it is a desirable feature that the reference system to which proper motions are referred be inertial, or in the terminology of general relativity, quasi-inertial. Since it is doubtless that the Hipparcos sphere will rotate with respect to any materialization of the inertial frame, either dynamical or geometrical, additonal work will be needed to align it with the extragalactic frame. This is a prerequisite to any progress in dynamical astronomy based on the Hipparcos catalogue.

It is now anticipated that a new conventional inertial system attached to radio sources will shortly supersede the FK4 system. These radio sources are either quasi-stellar objects (quasars ) or galactic nuclei. The possibility of defining a quasi-inertial reference system with extragalactic radio sources is based on the assumption that remote galaxies and quasars have no global rotational motion. In fact the actual assumption is somewhat stronger than that, since taken for granted that quasars are at cosmological distances, they have no significant angular motion detectable within the current capability of astrometry. Kovalevsky (1989) points out that in the most defavorable case a quasar at $100 \mathrm{Mpc}$ with a transverse velocity reaching the speed of light, the proper motion would not be larger than 0.7 mas/year. A more realistic limit is obtained by matching the transverse velocity to the recession which yields a proper motion of 0.01 mas/year (Argue, 1989). A still more realistic constraint is placed by the measurement of the relative displacement of the two close-by (in angle) sources NRA0512 and 3C345 wich proves to be less than 0.02 mas/year (Bartel et al., 1986).

Unfortunately no quasar but one ( $3 C 273$ with a magnitude of 13 ) is optically bright enough to be seen by Hipparcos. Intermediate objects and observations will have to be used to realize the connection between the two reference frames. 
The practical realization of the inertial frame is handed over to the Very Long Baseline Interferometers which, rotating with the earth determine the sources declination with respect to the instantaneous Earth's axis of rotation and their right ascension by reference to a selected fiducial source or some weighted average over many sources. At present the accuracy of radio sources position allows to materialize the extragalactic frame with a typical accuracy of 1 mas for about 100 objects, only for declinations above $-40^{\circ}$ (Sovers et al. 1988, Ma et al. 1986 ). Though the number of sources is rather small, the system possesses the remarkable property of not deteriorating with time because the error in the proper motion is negligibly small.

The alignement of the Hipparcos system to the VLBI's will permit to increase considerably the accessibility to the inertial frame for optical astronomy. The number of objects in the optical catalogue will be about 1000 times larger than the content in radiosources in the VLBI catalogue. With an average of 2 to 3 such stars per square degree a Schmidt plate will contain about 70 to 100 Hipparcos stars for the determination of the plate constants.

\section{LINKING HIPPARCOS TO THE INERTIAL FRAME}

\section{1. General Principle}

Several methods must be developed according as the link is between the Hipparcos frame and a stellar frame or if one searches to make the connection with the dynamical frame. The case of comparing the Hipparcos sphere to the dynamical reference frame will no be discussed in this paper. Interested readers may find valuable information in Söderhjelm and Lindegren (1982), Lindegren (1987) and Morando (1987).

Let us restrict to the first case ; it splits into two broad categories :

- Some stars belong to the two catalogues, but are not referred to a common reference system. This will be the case for a handful of radiostars observed by both Hipparcos and VLBI. In the latter case they are referred to the VLBI extragalactic reference frame mentioned in Sec. 2 . Positions and proper motions are assumed to be available, along with a covariant matrix. The situation applies also to stars belonging to the proper motions survey of Lick and Pulkovo ( Argue , 1989).

- No object appear in common in the two catalogues. This case pertains to the quasars in the VLBI system, not seen by Hipparcos. In that case one must resort to dedicated additional observations capable of giving the position of Hipparcos stars relative to quasars (Froeschlé and Kovalevsky 1982). 


\section{2. Mathematical Procedure}

Consider the link by means of radio stars, which is independant of the availability of observations with the Hubble Space Telescope.

Let $\mathbf{X}_{\mathbf{i}}$ and $\mathbf{V}_{\mathbf{i}}$ be respectively the position and velocity vector of the $i^{\text {th }}$ common object given in the HIPPARCOS catalogue the components of which are projected in the HIPPARCOS frame. In the present context the velocity vector $\mathbf{V}$ does not take into account the radial velocity ; this is the velocity vector associated to the transverse displacement of the star. Let $Y_{i}$ and $W_{i}$ be the same for the radio stars in the extragalactic frame.

Note that there is a subtle difference on how one should deal with the position vectors and the velocity vectors:

- The two position vectors noted $X_{i}$ and $Y_{i}$ stand for the same mathematical vector and strictly speaking should be written with the same letter. The two notations are necessary to recall that they are projected on two different basis.

- The velocity vectors refer to two different vectors, velocity of an object measured from two different reference systems, which are projected on two coordinates systems. We have then good reasons to use specific notations in that case.

Model assumption : in the following the two reference systems are assumed to differ only by a global rotation, possibly time dependant. In short, one neglects any kind of regional error that might be present in either system, that is to say each catalogue is supposed to provide a perfectly consistent set of positions and proper motions. This assumption will be checked at the end of the link from a careful examination of the residuals, to detect possible inadequacy of the model. In case the assumption fails the only way to remedy would be to consider that the global rotation is only the first term of an othenwise more complex expansion.

Let $\mathbf{R}$ be the rotation matrix between the two frames at the time $t$; thus we have

$$
\begin{aligned}
& X_{i}=[R] Y_{i} \\
& V_{i}=[R] W_{i}+[\dot{R}] Y_{i}
\end{aligned}
$$

The first equation accounts for the change of reference system while the latter allows for the change of frame and coordinate system. The rotation matrix depends on only three parameters, namely the axis of rotation and the angle of rotation, while [ $\mathbf{R}$ ] needs three other parameters to be defined. 
Clearly one can restrict to infinitesimal rotations and neglect any term of second order in [R] and $[\dot{R}]$. The most general such rotation matrix has the following form

$\mathbf{R}=\left[\begin{array}{rrr}1 & \gamma & -\beta \\ -\gamma & 1 & \alpha \\ \beta & -\alpha & 1\end{array}\right]$

and is associated to the three direct infinitesimal rotations $\alpha, \beta, \gamma$ respectively about the $x, y, z$ axes. The sign convention is chosen so that a rotation of the triad is meant and not that of the vectors. The sphere is slowly rotating with respect to the inertial frame in such a way that one can expand $\alpha, \beta$ and $\gamma$ in the vicinity of the reference time to of the HIPPARCOS catalogue as

$$
\alpha=\alpha_{0}+\alpha\left(t-t_{0}\right) \quad ; \beta=\beta_{0}+\dot{\beta}\left(t-t_{0}\right) ; \gamma=\gamma_{0}+\gamma\left(t-t_{0}\right)
$$

Finally the observation equations for a particular star become

$$
X_{i}=\left[\begin{array}{ccc}
1 & \gamma_{0} & -\beta_{0} \\
-\gamma_{0} & 1 & \alpha_{0} \\
\beta_{0} & -\alpha_{0} & 1
\end{array}\right] Y_{i}
$$

for the position in the two catalogues and

$V_{i}=\left[\begin{array}{ccc}1 & \gamma_{0} & -\beta_{0} \\ -\gamma_{0} & 1 & \alpha_{0} \\ \beta_{0} & -\alpha_{0} & 1\end{array}\right] W_{i}+\left[\begin{array}{ccc}0 & \dot{\gamma} & -\dot{\beta} \\ -\dot{\gamma} & 0 & \dot{\alpha} \\ \dot{\beta} & -\dot{\alpha} & 0\end{array}\right] Y_{i}$

for the velocity vectors. The second matrix in (2) accounts for the induced velocity brought about by the time-dependant rotation.

In the above equations the subscript stands for the stars to be compared; The $\mathbf{X}_{\mathbf{i}}, \mathbf{Y}_{\mathbf{i}}, \mathbf{V}_{\mathbf{i}}$ and $\mathbf{W}_{\mathbf{i}}$ are known ( within observational errors) and the six rotation parameters are searched for. From a set of objects known in the two systems a least squares fitting yields the six unknowns of the rotation. However the above presentation is somewhat misleading, as it conveys the idea that the each stars will provide two vector equations amounting to six scalar observation equations. In fact only two independant scalar equations are availbale in (1) and another two in (2). Hence it is useful to rewrite the observations equations in slight different way so as to make the linear structure more apparent and retain only independant equations. Let $I$ and $b$ be the longitude 
and latitude of a star, $\mu_{l}$ and $\mu_{b}$ the proper motion in longitude $\left(\mu_{l}=\frac{\mathrm{dl}}{\mathrm{dt}} \cos \mathrm{b}\right)$ and latitude. The choice of a particular set of spherical coordinates is irrelvant in the following discussion ; right ascension and declination can be substituted straightforwardly for the longitude and latitude. We obtain after some algebraic transformations of Eqts. 1 and 2 the design matrix as,

$\left[\begin{array}{l}\Delta l \operatorname{cosb} \\ \Delta b \\ \Delta \mu_{l} \operatorname{cosb} \\ \Delta \mu_{b} \cos b\end{array}\right]=\left[\begin{array}{cccccc}\cos I \sin b & \sin l \sin b & -\operatorname{cosb} & 0 & 0 & 0 \\ -\operatorname{sinl} & \operatorname{cosl} & 0 & 0 & 0 & 0 \\ \mu_{b} \operatorname{cosl} & \mu_{b} \operatorname{sinl} & 0 & \frac{1}{2} \cos 1 \sin 2 b & \frac{1}{2} \sin 1 \sin 2 b & -\cos ^{2} b \\ -\mu_{1} \cos l & -\mu_{1} \operatorname{sinl} & 0 & -\sin l \cos b & \cos l \cos b & 0\end{array}\right]\left[\begin{array}{l}\alpha_{0} \\ \beta_{0} \\ \gamma_{0} \\ d \alpha / d t \\ d \beta / d t \\ d \gamma / d t\end{array}\right]$

where $\Delta I=I \mathrm{vlbi}-I$ hip and accordingly for the other variables. If the observations were perfect in both catalogues, the link would be achievable with one and a half star only : six relationhips for six unknowns. In practice about twenty stars will be available to carry out the adjustement. The observation matrix will be a $4 n \times 6$ matrix obtained by repeating the above matrix at different $\mathrm{I}_{i}$ and $b_{i}$. One should note that the parameter $\gamma$ is determined only through $\Delta l$ (the latitude remains unchanged by a rotation about the z-axis) and $d \gamma / d t$ affects only the proper motion in longitude. As stated before the signs are such that we go from the Hipparcos frame to the extragalactic frame by direct rotations about the coordinate axes.

The left-hand side column contains the random vector of the observations. It appears clearly that the noise in each equations is a combination of the errors in both the VLBI and Hipparcos catalogue. Typically we have, for example in the first equation,

$\sigma^{2}(\Delta l \cos b)=\sigma_{\text {hip }}^{2}+\sigma^{2}$ vlbi

which shows that the two catalogues play a symmetric part in the achievement of the accuracy of the link. Because of the quadratic nature of this combination the final accuracy is determined primarily by the less accurate catalogue. This will be critical for the determination of the motion of the Hipparcos sphere with respect to the inertial frame, as it can only be obtained through the comparison of proper motions. One should remark that not every piece of information need be used to determine the matrix of rotation and its first derivative. Good positional information can be processed separately with the first two equations (for each star) of the design matrix to determine the rotational shift between the two systems at the epoch. Afterwards one will select 
stars with reliable proper motions to solve for the derivatives $\alpha, \beta, \gamma$. These proper motions could originate from various sources, in addition to radio stars program.

With the help of the design matrix it is easy to evaluate the accuracy to be obtained in the estimation, at least for a regular distribution of the linking stars. By neglecting the off-diagonal terms in the normal matrix one obtains from the variances when $\mathrm{N}$ stars are used for the link,

$\sigma(\alpha)=\sigma(\beta)=\sigma(\gamma)=\frac{\sigma}{N^{1 / 2}} \cdot \frac{1}{\left\langle\cos ^{2} b\right\rangle^{1 / 2}} \sim \frac{1.4 \sigma}{N^{1 / 2}}$

where $\sigma$ is the standard deviation of $\Delta l \cos b$ and $\Delta b$ ( Eq.3). For the derivatives we have

$\sigma(\dot{\alpha})=\sigma(\dot{\beta})=\sigma(\dot{\gamma})=\frac{\sigma^{\prime}}{N^{1 / 2}} \cdot \frac{1}{\left\langle\cos ^{4} b\right\rangle^{1 / 2}} \sim \frac{1.7 \sigma^{\prime}}{N^{1 / 2}}$

where $\sigma^{\prime}$ is the standard deviation of $\Delta \mu_{i} \cos \mathrm{b}$ and $\Delta \mu_{\mathrm{b}} \cos \mathrm{b}$. This crude evaluation is in perfect agreement with the monte carlo simulation presented in the next section.

\section{3 . 3 . Preliminary Results}

A simulation has been set up by Froeschlé and Kovalevsky (1982) with the nominal accuracy expected from the Hipparcos phase study. They have solved the equations taken in the form (1) and (2), apparently without introducing the fact that $|X|=|Y|=1$. It is difficult to assess how the use of redondant equations affects their results, although I am confident that their figures are representative of what would be obtained with indepedant equations. The formal standard deviations are probably slightly underestimated.

For radio stars observed in both systems they have found with the Hipparcos and radiosources astrometry of comparable accuracy ( $\sim 0.002$ in position ) that the initial rotation is properly recovered with an accuracy of

$$
\sigma=0.0035 / N^{1 / 2}
$$

where $\mathrm{N}$ is the number of stars in common. As for the rotational motion of the Hipparcos sphere the error is given by

$$
\sigma=0.005 / N^{1 / 2} \text { per year. }
$$

The reader is referred to their paper for a more detailed discussion of the results and the extension of the procedure to the link with the HST from the observations of quasars. To conclude this section it appears that a set twenty well distributed stars, with good VLBI positions and proper motions ( $\sigma \leq 1 \mathrm{mas}$ and $1 \mathrm{mas} / \mathrm{yr}$ ), and free of regional errors should be sufficient to perform the link at the millisecond level. 


\section{LINKING STARS}

The objects to be used to link the Hipparcos catalogue to the extragalactic frame are derived from proposals made by individuals and accepted by a Selection Committee. The VLBI frame was initially thought purely as a radio frame with no particular attention to optical observation (Argue, 1989 ). The first report of possibe detection of radio-emission by stellar sources was made in the mid-60's for Betelgeuse. A recent compilation given by Wendker (1987) contains about 300 radio stars covering a wide variety of emitters, either steady thermal or highly variable non thermal. The spectral type and optical properties are also very different.

A working group was appointed by IAU in 1978 to select objects suitable for both optical and radio observations. The first list contained 234 sources and was published by Argue (1984). Only object brighter than $V=17$ mag were retained, a convenient limit for the HST, but not for Hipparcos whose limitation is about $V=12.5$ mag.

Most objects are Galactic radio stars having a very small angulardiameter. Typical stars are RS CVn binaries, UV Cet, early-type emission stars and water and SiO masers. About 200 such stars in existing catalogues would be suitable provided that accurate VLBI position and proper motions could be obtained. Several campaigns were undertaken to detect radio stars with VLBI and ascertain their position at different epochs. Table 1 below summarizes the attempts made until 1986 to catch the stars ; the last two colums show strikingly that pointing at a known radio star is not the same as recording it.

Table 1. Mark III VLBI campaigns of radio stars, ( Lestrade et al. 1987a).

\begin{tabular}{ccccc}
\hline Years & $\begin{array}{c}\text { Frequency } \\
(\mathrm{GHz})\end{array}$ & $\begin{array}{c}\text { Number of } \\
\text { Stations }\end{array}$ & $\begin{array}{c}\text { Number of } \\
\text { stars } \\
\text { observed }\end{array}$ & $\begin{array}{c}\text { Number of } \\
\text { stars } \\
\text { detected }\end{array}$ \\
\hline & & & & \\
1982 Dec & 8.4 & 4 & 12 & 5 \\
1983 Feb & 1.7 & 3 & 2 & 2 \\
1983 Mar & 8.4 & 5 & 15 & 5 \\
1983 May & $2.3 / 8.4$ & 5 & 12 & 3 \\
1983 Jul & 5.0 & 6 & 12 & 7 \\
1983 Oct & 1.7 & 6 & 12 & 4 \\
1984 Mar & 5.0 & 4 & 1 & 1 \\
1984 Oct & 5.0 & 5 & 1 & 1 \\
1985 Mar & 8.4 & 3 & 6 & 0 \\
1985 Jun & 5.0 & 6 & 1 & 1 \\
1986 Mar & 10.8 & 4 & 3 & 2 \\
& & & & \\
\hline
\end{tabular}


At present ten radiostars have been monitored by VLBI during their radio outburst with the standard VLBI technique of bandwidth synthesis with sensitivity 10 to100 mJy used for astrometry on extragalactic sources. Positions at a first epoch and multiple epochs for some starshave been reported with various accuracies by Lestrade et al. (1988a and 1988b). ( see Table 2). The accuracy is mainly noise limited owing to the low flux density produced by radiostars compared to extragalactic sources. The best observed meet the precision requirement necessary to achieve the link without loss of accuracy.

Table 2. VLBI position of ten radio stars

\begin{tabular}{cccccc}
\hline Star & $\begin{array}{c}\text { Observation } \\
\text { epoch }\end{array}$ & $\begin{array}{c}\alpha(\mathrm{J} 2000) \\
\text { deg. }\end{array}$ & $\begin{array}{c}\text { RMS } \\
0.001\end{array}$ & $\begin{array}{c}\delta(\mathrm{J} 2000) \\
\text { deg. }\end{array}$ & $\begin{array}{c}\text { RMS } \\
0.001\end{array}$ \\
\hline LS161303 & 1983.789 & 40.1320250 & 120 & 60.2293222 & 70 \\
Algol & 1983.567 & 47.0422117 & 4.5 & 40.9556553 & 5 \\
UX Ari & 1983.567 & 51.6472396 & 3 & 28.7155628 & 5 \\
HR 1099 & 1983.215 & 54.1971833 & 3 & 0.5885167 & 20 \\
HR 5110 & 1983.567 & 203.6987054 & 3 & 37.1824608 & 2 \\
\% Cr B & 1983.567 & 243.6716713 & 10 & 33.8590194 & 10 \\
Cyg X1 & 1983.567 & 299.5903350 & 6 & 35.2016353 & 9 \\
SZ Psc & 1983.567 & 348.3490188 & 8 & 2.6753639 & 40 \\
HD26337 & 1987.01 & 62.4202667 & 70 & -7.8932333 & 7 \\
HD77137 & 1987.03 & 134.9281875 & 30 & -27.8161417 & 30 \\
& & & & & \\
\hline
\end{tabular}

A new and very sensitive VLBI technique based on phase reference to a powerful and angularly close extragalactic source has been developed lately (Lestrade et al. 1987b). It has yielded a relative position of Algol with an accuracy of 0.5 mas, obtained during its quiescent phase of emission when the density flux is as low as $3 \mathrm{mJy}$. This technique is now routinely used at the millisecond level to monitor positions of radiostars and multiple epoch positons are now available for five stars. It is expected that additional positions and proper motions of such stars will be measured with VLBI by the time the Hipparcos catalogue is completed.

In term of sensitivity this new method allows VLBI measurements to match closely that of the VLA. However with the VLA faint radio stars are observed with an accuracy of 0.01 arcsec at the moment, not sufficent to carry out a valuable link. Further modifications in the VLA configuration and programs will hopefully give by 1994 proper motions and parallaxes of 20 stars with declination $>30$ deg to the milliarcsecond level ( Johnston et al. 1988). 
For the link through HST and quasars, 173 stars near 92 extragalactic objects ( QSOs, BL Lacs and a few AGNs ) have been identified. These stars are bright enough to be observed by Hipparcos and are included with a high priority flag in the Input Catalogue (Hemenway et al. 1988). The relative postion of the star with respect to the fixed quasar will be monitored at different time so that the proper motion can be determined. The difference between the HST and Hipparcos proper motion is the result of the time dependant rotation of the Hipparcos sphere relative to the extragalactic frame. The expected accuracy of 2 mas rms, is an order of magnitude better than the current accuracies obtained by ground-based optical techniques ( Argue, 1989)

Closely connected to this work is the comparison between the radio positions and the optical positions obtained from meridian observations. VLBI positions are found to be comparable to optical positions although the differences are not significant below 0.05, the best precision obtainable with the automatic meridian circle of Bordeaux (Requième, 1973). An important point is to assess by how much the optical and radio photocenter are apart from each other. As the emission mechanism is poorly known it is of nearly no help in this regards; however VLBI mappings indicate that the source size is of the order of 1 mas which is also the upper bound of the separation between the radio and optical photocenter.

\section{CONCLUSION}

Hipparcos was launched on 8 August 1989 from Kuru in French Guiana, but the failure of the apogee boost motor prevented from putting the satellite into its geostationary orbit. A degraded and probably shortened mission has started anyway. We do not know at the time of writing what the mission lifetime will be, as it is influenced by such unpredictable parameters like the number of solar flares and the induced changes in the Van Allen belts. A link with the extragalactic frame would be valuable provided the accuracy in the retrieval of star positions and proper motions by Hipparcos is sufficiently close to the nominal accuracy of few mas; otherwise one should limit the ambitions to an adjustment to the FK5 frame, with a definition of 10 mas.

\section{ACKNOWLEDGMENTS}

J.F. Lestrade is gratefully acknowledged for his thorough reading of a preliminary version of this paper. Many of his remarks on the VLBI measurements have been incorporated in the final version.

\section{REFERENCES}

Argue A.N. , 1989. In the Hipparcos Mission, Vol. II, Chap . 16, ESA SP 1111.

Bartel et al. 1986. Nature, 319, 733. 
Froeschlé M., Kovalevsky J. , 1982. Astronom. \& Astrophys. 116 , 89.

Hemenway P.D., Benedict G.F., Jeffreys W.H., Shelus P.J., Duncombe R.L., 1988. Proc. Sitges Coll. "Scientific Aspects of the Input Catalogue Preparation" P. 461. Torra and Turon C. eds.

Johnston K.J., Florkowski D. ,Vegt C. de, 1988. Proc. Sitges Coll. "Scientific Aspects of the Input Catalogue Preparation" P. 447. Torra and Turon C. eds.

Kovalevsky J. , (1984). Space Sci. Rev. 39, 1.

Kovalevsky J., 1989. In Reference Frames in Astronomy and Geophysics, chap. 1. Kovalevsky, Mueller \& Kolaczek eds, Kluwer.

Kovalevsky J., Lestrade J.F., Preston R.A. .,1989. In the Hipparcos Mission, Vol. III, Chap . 17, ESA SP 1111.

Lestrade J.F., Preston R.A., Requième Y., Rappaport M., Mutel R.L., 1985. Proc. Aussois Coll. "Scientific Aspects of the Input Catalogue Preparation" P. 251. Perryman M. \& Turon C. eds.

Lestrade J. F. , Preston R. A. , Niell A. E. , . 1987a, in Proc. of the Third F.A.S.T. Thinkshop, P. 383. Bernacca P.L. \& Kovalevsky J. eds.

Lestrade J. F., Rogers A. E. E. , Niell A. E. , Preston R. A. . 1987b, in The Impact of VLBI on Astrophysics and Geophysics. IAU Sympos. 129, Cambridge, Mass. May 10-15.

Lestrade J.F. , White G.L. , Jauncey D. L., Preston R.A. , 1988b. Proc. Sitges Coll. "Scientific Aspects of the Input Catalogue Preparation" P. 481. Torra and Turon C. eds.

Lestrade J. F., Niell A. E. , Preston R. A., Mutel R. L. . 1988a, Astron. Jal. , 96, 1746.

Lindegren L. 1986 . In Proc. of the Third F.A.S.T. Thinkshop, P. 285. Bernacca P.L. \& Kovalevsky J. eds.

Ma C. et al. 1986. Astron. Jal. , 92,1020.

Requième Y., 1973. Astronom. \& Astrophys. , $23,453$.

Söderhelm S. , Lindegren L., 1982 . Astronom. \& Astrophys. , 110, 156.

Sovers O.J. , Edwards C. P. , Jacobs C.S., Liewer K.M., Treuhaft R. M. , 1988. Astron. Jal., In press.

Wendker H. J. , 1987. Astronom. \& Astrophys. Sup. Ser. , 69, 87. 